your funds. We are concerned if, through incompetence or laziness or ignorance, you can't lay hands on accurate raw data."

As for how investigations into scientific misconduct could be improved at the university level, that can be summed up on one word: consistency. "Each university has its own policy for how it investigates research fraud and misconduct, and it's a real dog's breakfast," said David Robinson, executive director of the Canadian Association of University Teachers. "There should be some central office, like the Office of Research Integrity in the United States, that applies consistent rules across the country, and applies some transparency and accountability." — Roger Collier, CMAJ

CMAJ 2015. DOI:10.1503/cmaj.109-5171

\title{
Can a new flu vaccine cure efficacy woes?
}

$\mathrm{N}$ ew influenza vaccines that for the first time protect against four strains of the virus will be widely available across Canada this year.

Each year, the World Health Organization predicts which strains are likely to dominate during flu season and drug companies make vaccines to match. For decades, these vaccines covered only three strains of influenza: two A subtypes and one B subtype. But since the 1980s, the influenza B virus has split into two distinct lineages making it harder to match. Between 2001 and 2013, seasonal flu vaccines covered the wrong B virus 7 out of 12 times.

New four-strain vaccines, which protect against an additional influenza $B$ subtype, may provide broader protection. "The strains of influenza that are circulating keep changing their coats, or the buttons on their coats, and a quadrivalent vaccine gives us more chances to match these changes," explains Dr. Noni MacDonald, an infectious disease expert and professor of pediatrics at Dalhousie University in Halifax.

The consequences of a poor match can be serious. The Public Health Agency of Canada estimates up to a quarter of hospital admissions and deaths are attributable to influenza B in any given season.

Quadrivalent flu vaccines were licensed in Canada last year, but this season will be the first time the new vaccines are widely used in public immunization programs. Provinces and territories have ordered about 12 million doses of flu vaccine, 2.9 million of which will be four-strain products.

Some jurisdictions, including Manitoba, the Atlantic provinces and the Yukon, will provide quadrivalent vaccines to all residents aged six months and older. Other public immunization programs, including those in Alberta

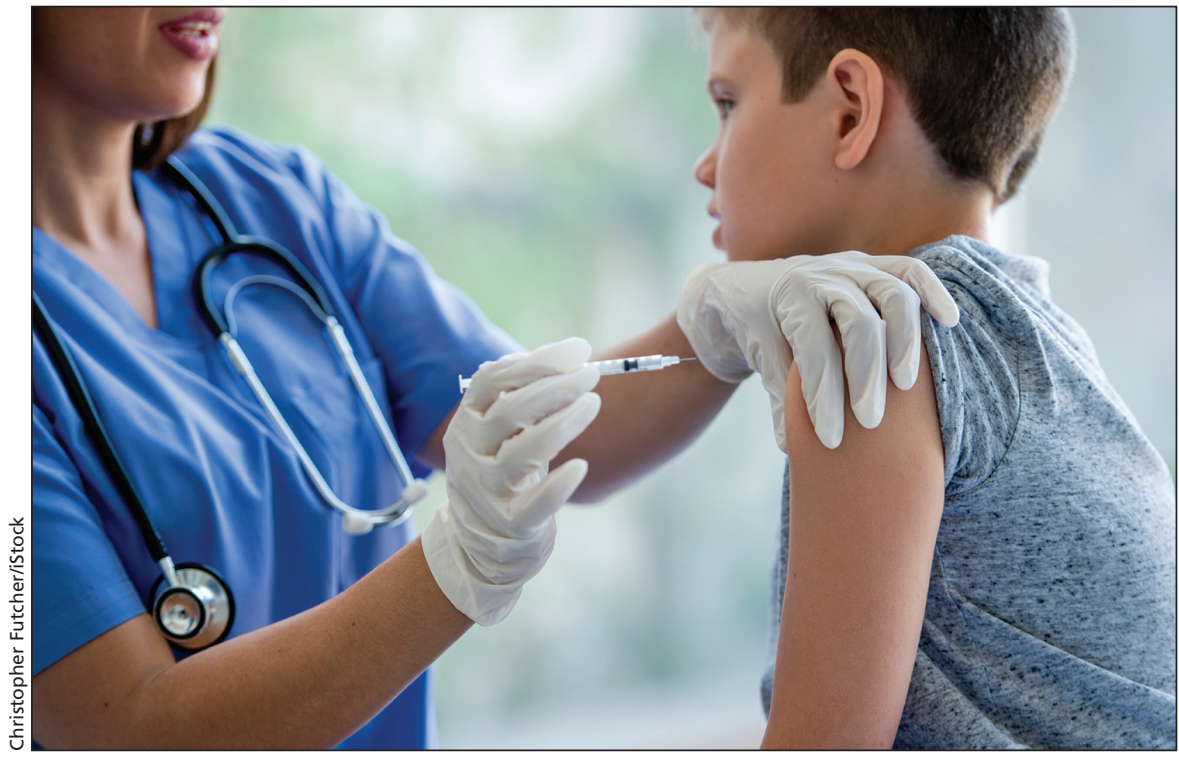

Canadians have a new weapon in their anti-flu arsenal this season.

and Ontario, will only provide fourstrain vaccines to children aged 6 months to 17 years. Most adults will continue to receive three-strain products.

"This mix of vaccines gives us a cost-efficient way to target the right levels of protection for our most vulnerable citizens," says Carolyn Ziegler, a spokesperson for Alberta Health.

The sticker price of quadrivalent flu vaccines can be 1.5 times higher than for trivalent products, but governments often negotiate lower prices with manufacturers behind closed doors. Studies also indicate that four-strain vaccines may still be more cost-effective overall, even at a higher price per dose, because of the broader protection they provide.

According to Canada's National Advisory Committee, both quadrivalent and trivalent flu vaccines are acceptable choices for adults, although children should receive four-strain products when available.

This recommendation is partly based on an expectation that adults will have some cross-protection from previous exposures to influenza B, says Dr. Dion Neame, senior director of scientific and medical affairs for vaccine manufacturer Sanofi Pasteur.

MacDonald notes that availability of a vaccine can influence recommendations. For example, the United States Centers for Disease Control and Prevention haven't favoured quadrivalent vaccines over three-strain products partly because they don't want people to miss getting the flu shot while waiting for access to a limited supply.

Receiving the trivalent vaccine, even in seasons when it is less than $23 \%$ effective, is still better than nothing, MacDonald notes. "Year after year, data from many different countries and many different settings show that people who get vaccinated have lower morbidity and mortality, particularly among the elderly and hospitalized kids." - Lauren Vogel, CMAJ

CMAJ 2015. DOI:10.1503/cmaj.109-5179 